\title{
1. Outline of the research project
}

\section{HIGH-TECH COMPANIES}

This book describes the organizational practices in the knowledge worker workplace and focuses on employees in the high-tech industry, or, to be exact, the IT industry (in some cases engineers developing the latest hardware, but mostly programmers). Even though it uses the word "work" more often than "labor", in line with other publications on the subject (this careful avoidance in the literature is most interesting and could be a topic of a separate study in itself), it describes broadly the current proletarianization of knowledge-intensive professions, using the example of software engineers.

The programming industry, quite surprisingly, is rather difficult to define. It encompasses both companies that offer sophisticated solutions (i.e. complex IT systems to target specific problems of organizations) and also other companies developing ready-made software to be sold on the mass market or quasi-mass market, usually without the possibility of modifications.

The degree of innovation in the programming market can also vary widely. Some companies focus on technological breakthroughs and the development of new solutions, but, there are also others that do repetitive implementations of ready-made IT systems, using commonly known tools, or offer simple IT services, specializing in cost-effectiveness, sales and marketing (particularly in the server-hosting market).

The typical division of the IT industry into hardware, software and services is very often blurred - many manufacturers of the latest equipment will also develop software and implement solutions; software producers successfully enter the hardware and services market; and service companies will offer software and hardware under their own brand names.

This confusion is increased by the lack of a consistent, coherent and precise business terminology. It can be difficult to find clear criteria to classify companies specifically within the high-tech industry. According to the Dictionary of Finance and Investment Terms, "high-tech stock" is simply (Downes and Goodman, 2006) 
"Stock of companies involved in high-technology fields (computers, internet related businesses, semiconductors, biotechnology, robotics, electronics). Successful high-tech stocks have above-average earnings growth and therefore typically very volatile stock prices.”

In this sense, the industry is very broad in its scope. On other hand, the AeA $^{1}$ definition, compiled in 2002 for use in statistical analysis, covers only three areas of the basic operation. In AeA's own words (AeA, 2002), it includes "high-tech manufacturing, communications services", and software and computer-related services. It does not include broad categories if the high-tech portion does not represent a clear majority. Also, AeA's definition does not include many "related" industries, such as biotechnology, engineering services, and research and testing services.

Krzysztof Klincewicz (2006), in his analysis of the lack of precise typologies, points out that in the literature there are three distinctive approaches to defining the high-tech industry. It is based on:

- the analysis of the social potential, in which the major criterion is the quantitative ratio of the employed engineers and scientists to the rest of the employees;

- the financial indicators, in which the criterion is the ratio of the $R \& D$ expenditure to the value of sales; and

- the assessment of the degree of technological and IT advancements with respect to others within the industry.

Because this book focuses on the social issues related to critical management studies, the most important of these approaches is the first. However, it should be noticed that the organizations studied, both Polish and American, do meet all three criteria of a high-tech company. ${ }^{2}$ All have been involved in creative work as well, that is, the development of their own software (and in one case their own hardware too) and implementations that are based mainly on their own ideas, though of course also using ready-made tools and modules for that process.

\section{THE HIGH-TECH INDUSTRY IN POLAND AND THE US}

This book is the result of a qualitative research project lasting several years but includes also some basic quantitative information relating to high-tech industry in Poland and the US that should better present a general picture of the studied industry. Such data constitute the background that influences the employment situation and the human relations 


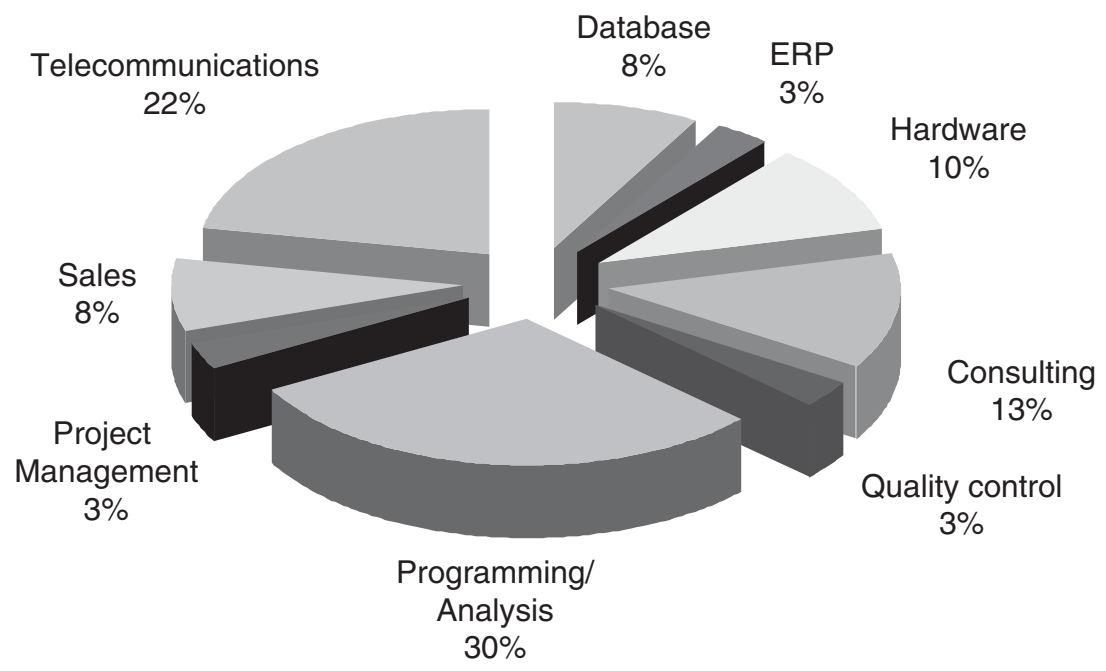

Source: QPracy.pl (2008).

Figure 1.1 Composition of IT industry job advertisements in Poland

and organizational practices within high-tech companies. Such quantitative data are not however a key element in the study here presented, as it focuses primarily on the social aspects of knowledge-intensive work. Most of the field data are drawn from the years just before the financial crisis of 2008, since 2009-11 may not have been fully representative.

According to the IT market research agency DiS (Anam, 2008), 100 of the top Polish companies spent over US\$2 billion on IT in 2007; five years earlier (2002) that figure was US\$1.5 billion. The value of the IT industry was estimated by DiS in 2007 to be nearly US\$8 billion. Similarly, according to analysts at PMR Ltd the value of the industry amounted to US\$8 billion in 2007 and was estimated to exceed US\$7 billion in 2008 (PMR, 2008). In 2007, even though the currency crisis in the US blurred the picture, growth was significant, reaching between 11 and 20 per cent (Frydrychowicz, 2008). 2009 was the first year in the last 20 that the value of the Polish IT market decreased by roughly 10 per cent (Maciejewski, 2010).

The shortage of IT specialists in Poland was estimated at about 10000 in 2008, and according to the infopraca.pl website, 10 per cent of the total job ads were for IT specialists (QPracy.pl, 2008), 30 per cent of them programmers (Figure 1.1). Both in Poland and worldwide, IT specialists are among the best-paid of professionals, together with managers, financiers and marketing specialists (Computer World, 2008). 
Table 1.1 Numbers of new jobs in high-tech in the US

\begin{tabular}{lcccc}
\hline 2005 & 2006 & 2007 & 2008 & 2009 \\
\hline 87400 & 139000 & 91400 & 77000 & -245600 \\
\hline
\end{tabular}

Source: TechAmerica's Education Foundation $(2008,2010)$.

A parallel situation exists in the US: salaries in the new-technology sector are higher by 87 per cent than the general company average (TechAmerica's Education Foundation, 2008). American new technology industry employed 1.6 per cent more people in 2007 than in 2006 (TechAmerica's Education Foundation, 2008), and the largest increase took place in the programming sector, with 82000 new employees. The number of high-tech employees amounted to 5.9 million in total (with no major change in 2008). These employment dynamics decreased in 2006 (Table 1.1), but still remained high. Unemployment in the high-tech industry in the US was low and did not exceed 2 per cent in 2007. The year 2009 brought a significant decline of 245600 jobs, but the 4 per cent decline was still lower than the 5 per cent in the private sector as a whole (TechAmerica's Education Foundation, 2010).

In 2008 , in the software services industry in particular, there was an increase of 86200 posts. In 2009, after five consecutive years of growth, there was a decline of 20700 jobs. It should be noted, though, that the fourth quarter of 2009 revealed a 10100 in employment growth in this industry, signaling a possible change from the downturn (TechAmerica's Education Foundation, 2010).

Before the crisis, venture capital investments in the industry in 2007 in the US increased by 6 per cent and amounted to almost US $\$ 17$ billion (TechAmerica's Education Foundation, 2008). High-tech companies' investment in $\mathrm{R} \& \mathrm{D}$, according to the same report, also rose by 6 per cent over the previous year and amounted to US\$74.9 billion in 2006.

The shortage of IT specialists in the US was one of the main reasons behind the increase on the limit of H-1B visas (for temporary, nonimmigrant workers) made by the American Congress in 1999-2003 195000 at the peak (Goodsell, 2007). Though the limit has gone down since 2004, it remains at 65000 annually.

It is thus clear that in both Poland and the US, high-tech industry is large and important, and that it operates under conditions of a constant need for highly qualified labor. The last circumstance produces a most interesting situation: programmers in many companies, both in Poland and in the US, have potentially, thanks to these conditions, a most favorable 
professional and organizational position - making their workplace a very interesting subject for qualitative analysis, since they are among the few who can challenge managerial domination.

\section{THE RESEARCH AREA}

The general research area of this book is the knowledge worker workplace in high-tech companies; specifically, it is a case study of Poland and the US.

Through a qualitative analysis of conceptual terms used by the social actors in the studied companies (as already mentioned, a precise description of the research methods is offered separately in Chapter 5), this book addresses a question: to what extent does work organization among knowledge workers have a democratic character? Even though managerial literature often points to, and even recommends, an egalitarian and democratic approach to that group of employees (Amar, 2002; Horibe, 1999; Newell et al., 2002), other research findings attest that the reality of work in high-tech companies, and in the high-tech workplace in particular, is quite different (Hochschild, 1997; Kunda, 1992; Perlow, 2003). That contradiction is the rationale for this project which aims to describe the workplace of knowledge workers in high-tech companies in detail and demonstrate its practical conditions on the basis of research "grounded" in field studies (Glaser and Strauss, 1967). This book approaches the task not by the use of some preconceptualized theoretical model, but through the study of these social phenomena, which are particularly important in the eyes of the population studied. The project focuses on perceptions of formalities and hierarchies, IT project schedules, the issue of trust in the high-tech workplace, and selected aspects of HRM and employee motivation.

The comparative analysis of the workplace in Polish and American companies does make a lot of sense. The US is the birthplace of IT and unquestionably a leader still in many high-tech areas, including programming. American universities are at the top of computer science departments' rankings, especially when measured by graduates' salaries. Find the majority of the computers worldwide are equipped with software (both applications and whole operational systems) developed in the US.

Poles, on the other hand, frequently win prestigious international programming competitions (including for example the TopCoder competition, the ACM International Collegiate Programming Contest, the Google Code Jam, the Microsoft Imagine Cup and the like); and the University of Warsaw has led the TopCoder.com ${ }^{3}$ rank for several years, 
and as of now (June 2011) is outranked only by Tsingua University and Tokyo University. Two other Polish universities have positions higher than the highest-ranked American one (currently MIT, although Carnegie Mellon for several years was higher). Polish programmers' aptitude and skills are unquestionable. For example, in the 2008 TopCoder edition, of the 49 participants, eight were Poles (PAIiIZ, 2008). Entry to this contest is restricted to beginners and the competition is one of the most prestigious in the world; its results have indeed greatly influenced Google's decision to open a research center in Poland (Bielewicz et al., 2006). There are about 150 other research centers in Poland, apart from Google's, and they were established by for example Credit Suisse, General Electric, IBM, Motorola, Intel and Siemens. The total investment value of these centers was estimated at $€ 5$ billion in 2006 (Nowaczyk, 2008). Also, in 2010 prestigious student internships at Microsoft in Redmont were dominated by Poles - as much as one-third of all intern are from Polish computer science departments. ${ }^{4}$

Despite this phenomenon, Polish companies currently can hardly be considered as leading any of the IT markets worldwide. Individual players enjoy local successes, and some international recognition - the development of such firms as Comarch, Sygnity and ADB is impressive, and the international expansion of Young Digital Planet or Gadu-Gadu obviously gives hope for change. Some companies slowly grow into recognition, for example Techland in 2009 was ranked at number 58 in the UK in the Develop 100 ranking of game producers, selling more games than for example a former star of this industry, Blizzard Entertainment. Also, Asseco (the biggest Polish IT company) is gradually going up the European rankings: for example in 2008 it was ranked number five in the "Top 100 European Software Vendors" (by Truffle Capital), with revenues of $€ 430$ million and as of now (June 2011) holds eighth position in the ranking, with 2009 revenues of 702 million euros.

Still, at present, there is an obvious disparity between the success of individual programmers in Poland, and Polish IT companies en masse. Also, no single off-the-shelf computer program originating in Poland has become a blockbuster.

According to some authors, improvement is only a matter of time. On 18 October 2006 two representatives of venture capital funds in Poland (Tomasz Czechowicz from MCI and Dariusz Wiatr from Hexagon Capital) made a public bet on whether by the end of 2008 a globally successful high-tech company would be established in the country (Domaszewicz, 2006). The chosen criteria were interesting: a presence either among the three popular European brands or within the top three European companies with respect to number of clients or market share. 
Possibly because of the global crisis the positive scenario did not come about. What makes matters worse for Polish companies in the long run, though, is that there are relatively few IT solutions developed in the Polish market that are also offered internationally. The success of Polish hightech giants still relies mainly either on the sale of Western solutions with only small modifications, or else on copying these solutions and/or adjusting them to local conditions.

Even the biggest success of a Polish IT firm is only recent, i.e. the game The Witcher, and it is not overwhelming. CD Projekt produced the game, and within the first three months sold 600000 copies. The game is distributed worldwide, and won awards in the industry magazines in 2007 (inter alia "Best PC RPG" by IGN, "PC RPG of the Year" by GameSpy, "Editor's Award" by PC Gamer, and others). Despite that success, in absolute terms, the game still lags behind the worldwide bestsellers. The Witcher II is in production, but even if it is more successful than the first version it will not dramatically change the picture.

One should be aware that in the computer entertainment industry, the market leaders are those firms that can produce not one best-selling title annually but a handful of them. In the year The Witcher premièred, CD Projekt's gross profit amounted to US\$3 million. Over a similar period, the market leader Electronic Arts made a gross profit of US $\$ 1.8$ billion. This is not to deny the success of CD Projekt of course, but at present it is difficult to say it is competing with the market leader in the computer games market. Moreover, other producers from both central and eastern Europe can show similar, if not bigger, achievements, for example, Croteam from Croatia (publisher of the Serious Sam game series). Still, with the Witcher 2 première in 2011 and very favorable reviews, there is a chance that CD Projekt will make sure Polish IT companies have had niche successes - other examples being Ivo Software (a multipurpose speech synthesis system) and Psiloc (cellphone software). There are also others, more effective in getting outsourcing orders - a good example here is Rinf from Wrockaw, the company established by a few former BP programmers, who now get contracts from Nokia, Siemens, Asseco or Comarch and have even opened a branch office in Silicon Valley. Nevertheless, global success is still missing, for many reasons. The first of these is the lack of a "business angel" type of financing, and the relatively small saturation by venture capital funds, while the second is the aversion of those entrepreneurs who have succeeded when investing their own capital in other ventures. In comparison, the German European Founders fund was established following the success of the Samwers brothers, who developed Jamba! (a big player in the cellphone software application development market) and have so far successfully invested in services as 
popular as, for example, LinkedIn.com, Facebook.com iLove.com, and the Polish Naszaklasa.pl.

Behind the failure of Polish companies to achieve a big break globally there lie also the disastrously poor support given by the government to the use of IT in the economy, the weak development of internet access and its high costs, ${ }^{5}$ a small basic market and the poor command of English among Poles, to mention just a few factors. Luck has also played an important role.

On the other hand, the success of neighbors from our region is striking. A team of programmers from Estonia was capable of developing Skype the most popular program for oral conversations on the internet, bought at the price of US\$2.6 billion by eBay in 2005 then sold to Microsoft for US $\$ 8.5$ billion in 2011. A team of Russian programmers created one of the most popular families of antivirus software used for broadly defined securities (Kaspersky); a Czech company produced AVG, one of five most popular antivirus programs; a team of programmers from Moldova originated The Bat!, a commercial email client alternative to Microsoft Outlook. These are just a few of the many examples.

It is not the purpose of this book to analyze the reasons for the lack of success of Polish off-the-shelf software producers, even if they are just organizational ones. However, the phenomenon does make Polish hightech companies look like an interesting research subject. Consequently, the analysis of the Polish software producers' workplace, its organizational practices and the perceptions of the employees is potentially important, especially in comparison with American companies. Study of both the similarities and the differences among knowledge-intensive organizations in both countries can play a part in furthering understanding of their specifics, and in producing a clear, precise analysis of the changes occurring in the workplace of knowledge worker worldwide.

For the purpose of this effort, the American portion of the research relies mostly on East Cost organizations and is accompanied by a series of interviews in different companies in similar locations, plus also a Silicon Valley start-up. ${ }^{6}$ Interestingly, the Silicon Valley start-up turned out to be the most difficult to gain access to, and I was not able to conduct observations there - thus the material from this company is quite limited and relies on only six interviews.

Still, Silicon Valley was not chosen as a main research area for this study for other reasons also. Numerous publications prove that the region is an exceptional social phenomenon (Saxenian, 1994), with a very specific work culture and management (English-Lueck, 2002) and with a habitat not seen elsewhere so far, that favor is the development of IT companies (Lee et al., 2000). Thanks to a cluster type of company 
development in that region (quite important for knowledge-intensive organizational development - see for example Nogalski and Grzybowski, 2007; Nogalski and Kowalczyk, 2008), and also to the active operations of a few of the most prestigious technical universities in the world, by collaborating closely with the largest latest-technology corporations an exceptional organizational environment and workplace for knowledge workers has been created (Bresnahan and Gambardella, 2004), with its own specific life cycle and development processes (Huggins and Izushi, 2007). Silicon Valley has also one of the highest concentrations of knowledge workers in the US: out of every 1000 employees in the private sector there, 286 work for high-tech companies (TechAmerica's Education Foundation, 2008).

Even though wide generalizations are not the purpose of this book, and the six organizations studied are not claimed to be the most representative of the whole industry, the uniqueness of Silicon Valley makes it especially difficult to use in international comparisons.

The whole research project was carried out over five years (2004-08), in two Polish ${ }^{7}$ companies based in Warsaw (PLOneos and PLSantos) and in three American companies (USVisualprog, USHuncor and USVird). The visits to the US took place in 2004-05, while the author was a Fulbright visiting scholar at Cornell University, Ithaca (NY), and in 2007-08, during a semester visiting research stay at Harvard University and a semester visiting research stay at the University of California, Berkeley.

The analysis is based on long-term, non-participant observations at PLOneos (four months) and USVisualprog (five months), and on interviews conducted at those firms. Research done in PLSantos, USHuncor and USVird was of a supplemental nature and was based on interviews with programmers and managers. In total 89 interviews were conducted. Auxiliary quantitative research was done during the observations, through a chronometric analysis of behavioral patterns at the workplace in PLOneos and USVisualprog and through mini-surveys.

The main part of the analysis focuses on knowledge-intensive workplaces' similarities to one another, irrespective of country, size or region. Individualities and differences are described less often and then are clearly emphasized. Organizational actors are not perceived as "dopes of their culture" and their nationality and national culture background is treated more as a discursive resource they draw from than as a fixed given-identity label (Barinaga, 2007).

Whenever in the text "both organizations" are mentioned, the reference is to PLOneos and USVisualprog. In turn, whenever the term "all of the studied organizations" is used, the research findings apply to both the research material gathered during observations and the interviews 
completed in all five companies. The organizations studied are described in detail in Chapter 5, in the section. "The Research Problem".

As the subject of this book is work in knowledge-intensive companies and organizational relations at the knowledge workers' workplace, it is necessary to discuss that basic terminology. The next three chapters, Chapters 2, 3, and 4, describe the work-related issues, the knowledge companies, and the term "knowledge workers" in the context of the sociology and management literature on employees. Chapter 5 describes the research methods and the methodological assumptions for this project. The Chapter 6 refers to the contemporary bureaucracy and starts the presentation of the research findings. Chapters 7, 8 and 9 cover the topics of trust in IT projects, and of the selectively chosen (as being important for the interlocutors) elements of HRM and motivation in knowledge work. Chapter 10, the last, summarizes the project through an interpretation of ideological control and the position of observed management practices in the classic dichotomy of the Theory $\mathrm{X}$ and Theory $\mathrm{Y}$ approaches.

\section{NOTES}

1. AeA, formerly known as the American Electronics Association, merged into TechAmerica (www.itaa.org) in 2009. Even before this merger it was the largest American organization in the electronics industry, with a membership of 2500 organizations, employing over $1.8 \mathrm{~m}$ people in total. It was established in 1943, and its main activities are the development of industry analysis, lobbying and the collective representation of its members.

2. A more detailed description of the companies studied, as well as a section on research methods, are included in Chapter 5.

3. Available at: http://www.topcoder.com/stat?c=school_avg_rating (accessed 16 May 2010).

4. As reported on http://www.emetro.pl/emetro/1,82493,7848960,Co_trzeci_praktykant_w_ Microsoft_pochodzi_z_Polski.html (accessed 15 June 2010).

5. In the Global Information Technology 2010-2011 rankings of "Networked Readiness", compiled by the World Economic Forum in cooperation with INSEAD, Poland was at 62, just below South Africa, and above Trinidad and Tobago. Among all European countries, Poland outstripped only Greece, Romania, Bulgaria and Slovakia. The higher positions in the rankings were occupied for example by Colombia, Vietnam and Oman hardly regarded, at least in Poland, as being significantly more developed countries. The full ranking is available on the internet at: http://www.weforum.org (accessed July 2010).

6. A more detailed description of the organizations analyzed is given in the Chapter 5 .

7. The names of all the studied companies are fictitious, as are the interlocutors' cryptonyms. Some details insignificant for the analysis have been changed, to disallow companies' identification, in line with the rules of the ethics of anthropology (Madison, 2005). 